\title{
Use of a mobile application for self-management of pancreatic enzyme replacement therapy is associated with improved gastro-intestinal related quality of life in children with Cystic Fibrosis
}

\author{
M. Boon ${ }^{\mathrm{a}, *}$, J. Calvo-Lerma ${ }^{\mathrm{b}}$, I. Claes ${ }^{\mathrm{a}}$, T. Havermans ${ }^{\mathrm{a}}$, I. Asseiceira ${ }^{\mathrm{c}}$, A. Bulfamante ${ }^{\mathrm{d}}$,

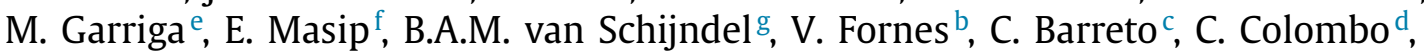 \\ P. Crespo ${ }^{\mathrm{b}, 1}$, S. Vicente ${ }^{\mathrm{e}}$, H. Janssens ${ }^{\mathrm{h}}$, J. Hulst ${ }^{\mathrm{g}, 2}$, P. Witters ${ }^{\mathrm{a}}$, R. Nobili ${ }^{\mathrm{d}}$, L. Pereira ${ }^{\mathrm{c}}$, \\ M. Ruperto ${ }^{\mathrm{e}}$, E. Van der Wiel ${ }^{\mathrm{h}}$, J.G. Mainz ${ }^{\mathrm{i}, \mathrm{j}}$, K. De Boeck ${ }^{\mathrm{a}}$, C. Ribes-Koninckx ${ }^{\mathrm{b}}$ \\ a Department of Pediatrics, Center for Cystic Fibrosis, University Hospital Leuven, Leuven, Belgium \\ ${ }^{\mathrm{b}}$ Instituto de Investigación Sanitaria La Fe, Valencia, Spain \\ ${ }^{\mathrm{c}}$ Associação para a Investigação e Desenvolvimento da Faculdade de Medicina, Lisbon, Portugal \\ dUniversità degli Studi di Milano, Fondazione IRCCS Ca" Granda, Ospedale Maggiore Policlinico, Milan, Italy \\ e Unidad de Fibrosis Quística, Hospital Universitario Ramón y Cajal, Madrid, Spain \\ ${ }^{\mathrm{f}}$ Gastroenterology and Pediatric cystic Fibrosis Unit, La Fe Hospital, Valencia, Spain \\ ${ }^{g}$ Department of Pediatrics, div of Gastro-Enterology, Erasmus MC-Sophia Children's Hospital, University Hospital Rotterdam, the Netherlands \\ ${ }^{\mathrm{h}}$ Department of Pediatrics, div Respiratory Medicine and Allergology, Erasmus MC-Sophia Children's Hospital, University Hospital Rotterdam, the \\ Netherlands \\ ${ }^{i}$ Cystic Fibrosis Center for Children and Adults, Jena University Hospital, Germany \\ j Cystic Fibrosis Center Brandenburg Medical School (MHB), University, Brandenburg an der Havel, Germany
}

\section{A R T I C L E I N F O}

\section{Article history:}

Received 20 November 2019

Revised 2 April 2020

Accepted 4 April 2020

Available online $\mathrm{xxx}$

\section{Keywords:}

Cystic fibrosis

Pancreatic insufficiency

PERT

Quality of life

PedsQL GI

CFAbd-Score

MyCyFAPP

Mobile application

\section{A B S T R A C T}

Background: Most patients with cystic fibrosis (CF) suffer from pancreatic insufficiency (PI), leading to fat malabsorption, malnutrition, abdominal discomfort and impaired growth. Pancreatic enzyme replacement therapy (PERT) is effective, but evidence based guidelines for dose adjustment are lacking.

A mobile app for self-management of PERT was developed in the context of the HORIZON 2020 project MyCyFAPP. It contains an algorithm to calculate individual PERT-doses for optimal fat digestion, based on in vitro and in vivo studies carried out in the same project.

In addition, the app includes a symptoms diary, educational material, and it is linked to a web tool allowing health care professionals to evaluate patient's data and provide feedback.

Methods: A 6-month open label prospective multicenter interventional clinical trial was performed to assess effects of using the app on gastro-intestinal related quality of life (GI QOL), measured by the CF-PedsQL-GI (shortened, CF specific version of the Pediatric Quality of Life Inventory, Gastrointestinal Symptoms Module).

Results: One hundred and seventy-one patients with CF and PI between 2 and 18 years were recruited at 6 European CF centers. Self-reported CF-PedsQL-GI improved significantly from month 0 (M0) (84.3, 76.4-

Abbreviations: APP, Mobile Application; BMI, Body Mass Index; CF, Cystic Fibrosis; CFA, Coefficient of Fat Absorption; CF-Abd Score, Cystic Fibrosis specific validated

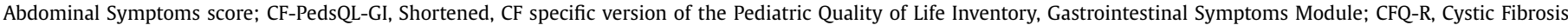

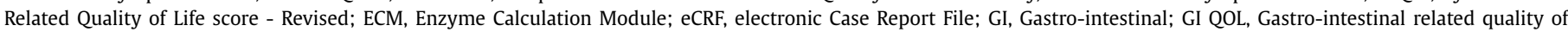

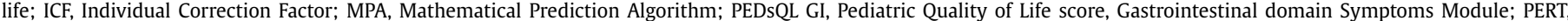
Pancreatic Enzyme Replacement Therapy; QOL, Quality of Life; TOD, Theoretical Optimal Dose; VAS, Visual Analogue Scale.

* Corresponding author.

E-mail address: mieke.boon@uzleuven.be (M. Boon).

1 Current address: Department of Health Science, European University Miguel de Cervantes, Valladolid, Spain.

2 Current address: Division of Gastroenterology, Hepatology and Nutrition, Hospital for Sick Children, Toronto, Canada. 
90.3) to month 6 (M6) $(89.4,80.35-93.5)(p<0.0001)$. Similar improvements were reported by parents. Lower baseline CF-PedsQL-GI was associated with a greater improvement at M6 $(p<0.001)$.

Conclusions: The results suggest that the MyCyFAPP may improve GI QOL for children with CF. This tool may help patients to improve self-management of PERT, especially those with considerable GI symptoms.

(c) 2020 European Cystic Fibrosis Society. Published by Elsevier B.V. All rights reserved.

\section{Introduction}

Cystic Fibrosis (CF) causes fat malabsorption and early onset pancreatic insufficiency (PI) in approximately $80-90 \%$ of patients [1]. Malnutrition and stunting can only be avoided by accurate supplementation with pancreatic enzyme replacement therapy (PERT) and close nutritional follow-up.

Inadequate PERT dosage leads to maldigestion and gastrointestinal complaints that can impact daily life [2].

However, there is little evidence about the optimal PERT dose and how to adapt it to individual patients [3-5]. This was reflected by the results of a European study in children with CF where similar nutritional parameters (weight, height and BMI z-scores) were found despite a high variability in PERT dose used in the different centers [6]. Compared to the respiratory aspects of $\mathrm{CF}$, little research focuses on improving gastrointestinal/nutritional treatment [4]. However, patients rank gastro-intestinal discomfort as very important and consider them as high research priority [7]. Furthermore, nutritional status is an independent predictor of survival [8]. The lack of an evidence-based tool to improve PERT, leads to low treatment efficacy: patients often base their PERT on habitual doses. If the dose is not properly adjusted, every single meal can contribute to discomfort and ultimately to malnutrition. In contrast, if dosing is optimal for every specific meal, nutrients will be properly digested, contributing to adequate nutritional status. Thus, optimal PERT control can have a positive impact on overall disease prognosis.

The scope of the Horizon 2020 project MyCyFAPP (grant number 643,806 ) was to develop and validate a tool, namely a mobile application (app), for patients with CF, to optimize and personalize their PERT doses with every meal and in real time [9]. We showed that the optimal dose for maximal lipid digestion is mainly dependent on food characteristics and developed a mathematical predictive algorithm (MPA) that establishes the optimal PERT dose based on food properties [10]. This was tested in a pilot study, resulting in a median coefficient of fat absorption above 90\% [11]. The PERT calculation method was implemented into the app to assist patients to optimize their PERT dose.

As the last part of the MyCyFAPP project, we study the impact of using the MyCyFAPP in real life on patients' gastrointestinal (GI) symptoms, quality of life (QOL), general wellbeing and nutritional status. We investigated demographic and biometrics and changes over time whilst using the app. This investigation is essential before implementation of the app in clinical practice can be considered. We hypothesize that QOL, general well-being and nutritional status improve after using the app for at least 3 months.

\section{Methods}

\subsection{Study protocol}

A 6-month, open label, prospective, multicentre interventional clinical trial was performed in stable children with $\mathrm{CF}$, between 24 months and 18 years of age, all having PI (fecal elastase $<200$ $\mathrm{mcg} / \mathrm{g}$ stool) and treated with PERT.

Children were recruited in 6 European CF centres participating in the MyCyFAPP project (Lisbon, Madrid, Milan, Leuven, Rot- terdam, Valencia). The diagnosis of $\mathrm{CF}$ was confirmed by a sweat chloride $\geq 60 \mathrm{mEq} / \mathrm{L}$ and/or the presence of 2 disease-causing mutations in the CFTR gene. Patients with acute infections or acute abdominal pain and patients who recently ( $<3$ months) started CFTR modulation therapy were also excluded.

The study protocol was approved by the Ethical Committees of all participating centres. Written informed consent was obtained from parents and from children over 12 years of age.

\subsection{Measures}

\section{QOL was evaluated using}

- The shortened, CF specific version of the Pediatric Quality of Life Inventory, Gastrointestinal Symptoms Module (CF-PedsQLGI): a GI specific QOL questionnaire, consisting of 9 subdomains and 54 questions for use in children 5-18 years of age and in parents of children $>2$ years. This shortened version of the PedsQL GI $[12,13]$ was validated for use in children with CF $[14,15]$. Lower scores indicate more severe/frequent symptoms.

- The Cystic Fibrosis Related Quality of Life score - Revised (CFQR) [16]: a CF specific questionnaire for general evaluation of QOL, for use in children $>6$ years and their parents. Only subscale scores can be calculated, lower scores indicating worse QOL.

- The Cystic Fibrosis specific validated Abdominal Symptoms Score (CFAbd-Score) [17-19]: a recently developed and validated questionnaire to evaluate GI QOL in CF. It consists of 28 items in 5 domains and can be used from 5 years on and in parents of children $>2$ years. Higher scores indicate more severe/frequent symptoms.

- A visual analogue scale (VAS): a linear scale to answer the question 'How is your health state today?' from $0 \%$ to $100 \%$.

Age-specific versions of the questionnaires were used in children and parents. (Supplementary file 1). All questionnaires were available in 5 languages (Dutch, Flemish, Italian, Portuguese, Spanish).

\subsection{Intervention}

Use of the mobile MyCyFAPP was implemented for a period of 6 months (Fig. 1). The mobile application ecosystem was developed in the context of the MyCyFAPP project by the technical partners SINTEF (Trondheim, Norway), Imaginary (Milan, Italy), Youse (Munich, Germany) and ITACA-Sabien (Polytechnic University of Valencia, Spain) $[9,20,21]$.

The developed tool has 4 main features (see Supplementary file 2-5): (a) a diary where patients can record meals to be eaten, so that an optimal PERT dose (both expressed as lipase units and number of pills in function of the used enzyme preparations) is recommended in real time as instructed by the MPA; (b) a symptoms diary where patients report on GI symptoms including pain, gastroesophageal reflux, nausea, stools consistency...; (c) a link to the professional webtool containing follow-up charts showing the evolution of nutrient intake and symptoms over time and enabling direct communication between patients and healthcare professionals (see Supplementary file 4 for more detailed information); and 

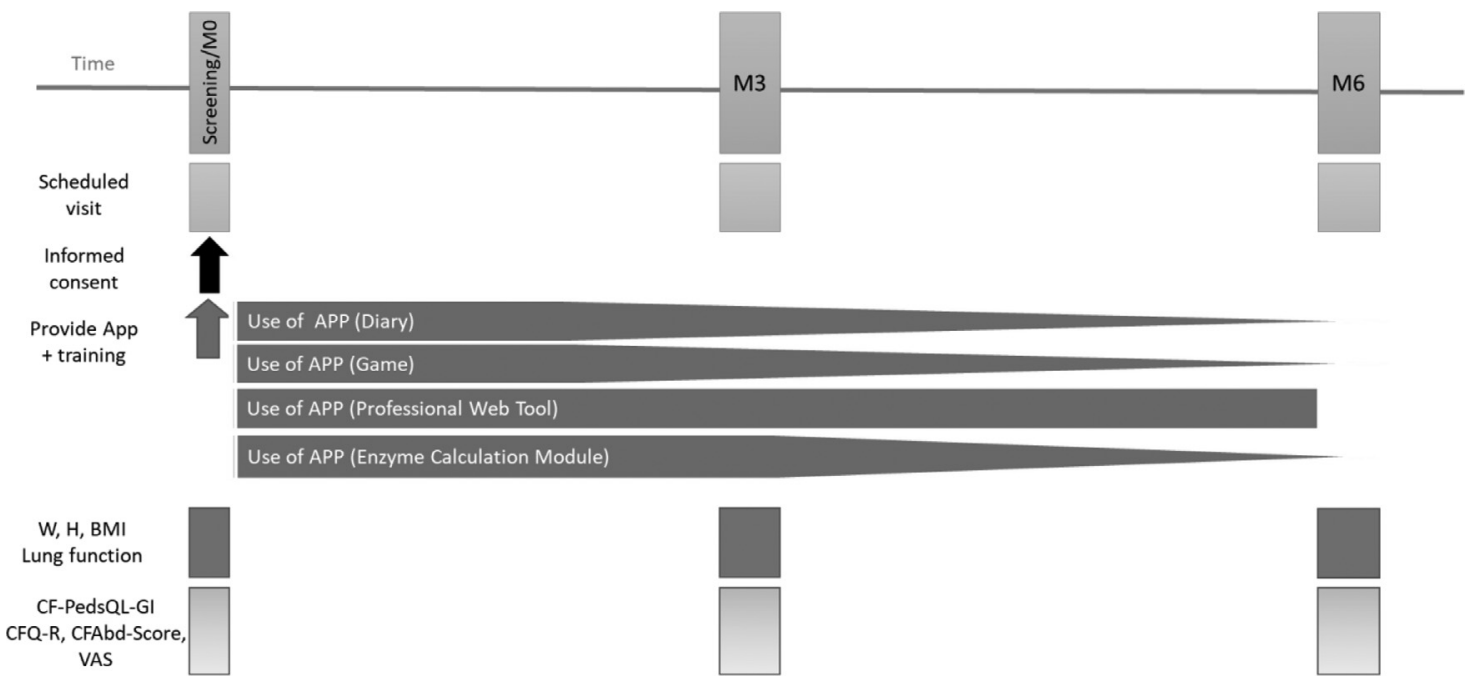

Fig. 1. Timeline of the study.

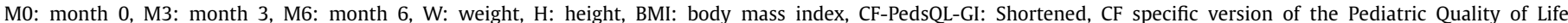

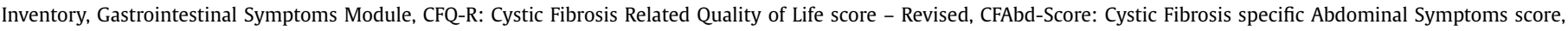
VAS: Visual Analogue Scale.

(d) educational material to improve the knowledge about nutrition in general and CF-specific conditions (a handbook, available for parents and children $>12$, and games, on a separate tablet for children $<12$ years).

All app content was available in 6 languages (Dutch, English, Flemish, Italian, Portuguese, Spanish). The translations from English language were performed by each clinical team at the participating CF units, including pediatricians and dietitians. Then backwards translation was validated with native speakers. All the contents of the app were adapted so participants aged 12 years and older could understand all the contents independently [20,21].

At the run-in visit standardised instructions about the use of all app features were given to patients and parents. Patients/parents were asked to use the app at least once daily during the first month of the trial, and then at least twice weekly. Patients and parents used the app (separately or together) in function of their own capabilities and interest. Entered data were reviewed at least once per week by a health care worker (mainly dietician or doctor) of the patient's CF center.

\subsection{Outcome parameters}

\subsubsection{Primary outcome}

Change in CF-PedsQL-GI was assessed at M3 and M6. Based on the validation results and assuming a 15\% drop-out, 200 patients would be needed to achieve a power of $80 \%$ and a significance level of 0.05 to see an improvement of 3.5 units in CF-PedsQL-GI, which was considered clinically meaningful, based on data from previous studies [15,22].

\subsubsection{Secondary and tertiary outcomes}

Secondary outcomes were change in weight z-score, height zscore, BMI Z-score (expressed according to CDC references [23]), PERT dose, lung function $\left(\mathrm{FEV}_{1}, \mathrm{FVC}, \mathrm{FEV}_{1} / \mathrm{FVC}\right.$ and $\mathrm{FEF}_{25-75}$, expressed as z-scores [24]), change in CFQ-R subscales, CFAbd-Score and VAS. These were assessed at M3 and M6. Weight and height were clinical assessments without standardized procedure. Anyhow, all height measurements were performed with a stadiometer and without shoes.

Dose of PERT was defined as the mean dose of PERT during one complete day, measured during 3 consecutive days. Patients were asked to register PERT doses during 3 consecutive days at M0, M3 and M6.

Percent days of app use (\% app use) was evaluated as tertiary outcome and calculated dividing the number of days PERT was registered by the total number of days in the study. Therefore, the goal for \% app use was set at $40 \%$ (at least once daily during the first month and then $2 x$ per week after that).

Patient characteristics (age, biometry, lung function) and questionnaire results were entered in an online electronic Case Report Form (eCRF). At the end of the trial, data from the app (PERT dose, $\%$ app use) were exported to the eCRF.

\subsection{Statistical analysis}

Data were summarized using mean (standard deviation) and median (1st, 3rd quartiles) for continuous variables and using absolute and relative frequencies in case of categorical variables. Spearman's or Pearson's correlation coefficient was calculated according to the type of relationship.

As all QOL questionnaires are scales, changes in CF-PedsQL-GI, CFQ-R subscales, CFAbd Scale scores and VAS were assessed by a mixed ordinal logistic regression model. Gender, age, baseline $\mathrm{FEV}_{1}$ z-score, baseline BMI $\mathrm{z}$-score and\% of app use were considered as co-factors in the multivariable regression models. The non-independence of data due to repeated measures per individual, was controlled extending the model with a random factor with random intercept ("Patient"), as well as the 'center' was corrected as a random effect. A sensitivity analysis using bayesian approach was applied to increase the certainty of the changes in CF-PedsQLGI from M0 to M6. Weak informative priors for the coefficients of the fixed effects were set at $\mathrm{N}(0,8)$.

BMI, Weight, Height and $\mathrm{FEV}_{1}$ z-scores were analysed by means of linear mixed regression models. Average PERT intake was logtransformed, to guarantee a normal distribution. Similar analyses were performed for changes in secondary endpoints.

As the intended total number of patients was not achieved (171 out of 200), a post-hoc power calculation was conducted (Montecarlo simulation, 10,000 iterations) to assess the statistical power with the observed effect. Analyses were performed using R software (version 3.5.2). 
M. Boon, J. Calvo-Lerma and I. Claes et al./Journal of Cystic Fibrosis $x x x$ (xxxx) $x x x$

Table 1.

Demographic characteristics at baseline expressed as median (1st and 3rd Q) or number and percentage $(n=171)$.

\begin{tabular}{ll}
\hline Age $(\mathrm{y})$ & $8.5(5.5,12.85)$ \\
Average PERT intake (LU/day) & $155,425(94,996240,000)$ \\
Average PERT intake (LU/kg/day) & $5659(3474,8108)$ \\
Weight z-score & $-0.28(-0.87,0.4)$ \\
Height z-score & $-0.26(-0.88,0.46)$ \\
BMI z-score & $-0.28(-0.86,0.37)$ \\
FEV 1 pp(\%) & $93.02(80.98,102.56)$ \\
FEV $_{1}$ Z-score & $-0.58(-1.52,0.22)$ \\
Gender & \\
Male & $88(51.46 \%)$ \\
Female & $83(48.54 \%)$ \\
Age group CF-PedsQL-GI category & \\
Toddlers 2-4y & $26(15.2 \%)$ \\
Children 5-7y & $40(23.39 \%)$ \\
Children 8-12y & $55(32.16 \%)$ \\
Teens 13-18y & $50(29.24 \%)$ \\
No. of children with self-report CF-PedsQL-GI \\
Toddlers 2-4y $(n=26)$ & 0 \\
Children 5-7y $(n=40)$ & $30(75 \%)$ \\
Children 8-12 y $(n=55)$ & $53(96 \%)$ \\
Teens 13-18y $(n=50)$ & $49(98 \%)$ \\
\hline
\end{tabular}

\section{Results}

\subsection{Demographics and clinical data}

We recruited 171 patients. Baseline characteristics are presented in Table 1. Twenty tree (13.4\%) patients (4 in Lisbon, 3 in Madrid, 5 in Valencia, 6 in Milan, 3 in Leuven and 2 in Rotterdam) dropped out during the study (20 due to adverse events, 3 did not want to continue the trial), so a total of 148 patients completed the trial at M6.

\subsection{Unadjusted associatons}

\subsubsection{Primary outcome: change in CF-PedsQL-GI}

There was statistically significant improvement of the median CF PedsQL-GI from M0 (84.3) to M6 (89.4) ( $p<0.0001$ ), but not from M0 (84.3) to M3 (85.2) ( $\mathrm{p}=0.095)$ (median 84.3-85.2, respectively, $p=0.095$ ) (Table 2 ). In parents, the change was statistically significant from M0 (80.1) to M3 (84.7) ( $<<0.001$ ), and sustained at M6 (85.7) ( $<<0.001)$ (Table 2).

Most subscores (7/9) of the CF-PedsQL-GI improved statistically significantly (Fig. 2, Supplementary file 6-7). There was a statistically significant correlation between scores from children and their parents (r 0.646, $p<0.001$ ).

App use was generally well accepted. Twenty adverse events occurred and led to interruption of the trial: 2 patients had symptoms possibly related to an excessively high PERT dose (hard stools, painful bowel movement, 1 appendicitis), 9 patients had symptoms possibly related to an excessively low PERT dose (loose, greasy or brilliant stools), 9 patients were hospitalized with respiratory exacerbation.

\subsection{Secondary and tertiary outcomes}

\subsection{1. $C F Q-R$}

There were small but statistically significant changes in a minority of CFQ-R subdomains in all age categories as indicated in Supplementary file 8-9. However, the statistically significant changes in the different domains were not necessarily seen in all age groups. This might be because there are real differences between the age groups or because the questions differ for the different age categories.

\subsubsection{Visual analogue scale (VAS)}

While there was no change in the median VAS scores over time, there was statistically significant improvement from M0 (median 90, IQR $(80,90)$ ) to M6 (median 90, $\operatorname{IQR}(80,100)$ ) in patients $(p=0.026)$, but not in parents (M0 median 85 , IQR $(80,90)$; M6 median 90, IQR (80-95), $(p=0.056)$ ) (Supplementary file 10).

\subsubsection{CFAbd-score}

For patients, CFAbd-scores (median, IQR) did not change from M0 (11.5 (6.97, 18.25)) to M3 (10.65 (5.7, 17.8)) (p 0.20), but improved statistically significantly at M6 $(9.9(4.8,15.6))(\mathrm{p} 0.005)$. CFAbd-scores for parents improved statistically significantly from M0 $(13.95(8.23,20.62))$ to M3 $(10.9(6.6,19.2))(\mathrm{p} 0.01)$ and M6 $(10.85(5.38,17.6))(p<0.001)$ (Supplementary file 11 and 12$)$.

\subsubsection{Biometry}

A statistically significant increase in height z-score occurred from M0 (median -0.26 , IQR $(-0.88,0.46)$ ) to M6 $(-0.12$, IQR $(-0.65,0.62))(\mathrm{p} 0.011)$. A statistically significant decrease in weight $\mathrm{z}$-score was found (M0 $-0.28,-0.87 ; 0.4$ versus M6 -0.32 , $-0.91 ; 0.26)$ ( $\mathrm{p}$ 0.005), resulting in a statistically significant decrease in BMI z-score (M0 -0.28 , IQR $(-0.86,0.37)$ versus M6 -0.41 , IQR $(-1.02,0.29))(p=0.005)$ (Supplementary file 13$)$.

\subsubsection{Percentage (\%) of app use}

The median\% of app use was $46 \%$ (IQR $27-76 \%$ ), which is in line with the anticipated use. The proportion of patients with a\% of app use above the target of $40 \%$ was $57.4 \%$.

\subsubsection{Change in dose of pert}

Overall PERT intake did not change: from a median of 5859 $\mathrm{LU} / \mathrm{kg} /$ day $(3704,8126)$ at M0 to $6079 \mathrm{LU} / \mathrm{kg} /$ day $(4271.7934)$ at M3 (effect size 0.046, CI95\% [-0.04, 0.13], $p=0.29$ ) and $6165 \mathrm{LU} / \mathrm{kg} / \mathrm{day}$ at M6 $(4160,7871)$ (effect size 0.069, CI95\% [-0.02, 0.16], $p=0.12$ ).

Table 2.

Results for CF-PedsQL-GI obtained from children and parents. Descriptives are presented as median (1st, 3rd Q). For each score, the estimated effect between M0 and M3 and between M0 and M6 from the ordinal regression model is presented as the Odds Ratio [95\% Confidence Interval] and the $p$-value.

\begin{tabular}{llll}
\hline Cf-PedsQL-GI questionnaire & M0 & M3 & M6 \\
\hline & Median (1st, 3rd Q.) & Median (1st, 3rd Q.) & Median (1st, 3rd Q.) \\
\hline CF-PedsQL-GI patients & $N=132$ & $N=123$ & $N=116$ \\
& $84.3(76.4,90.3)$ & $85.2(77.1,91.9)$ & $89.4(80.35,93.5)$ \\
OR, p-value & - & $1.45, p=0.095$ & $3.11, \boldsymbol{p}<\mathbf{0 0 0 1}$ \\
CI 95\% OR & & {$[0.94,2.25]$} & {$[1.95,4.94]$} \\
\hline CF-PedsQL-GI parents & $N=167$ & $N=156$ & $N=148$ \\
OR, p-value & $80.1(72.9,88.9)$ & $84.7(76.28,91.4)$ & $85.7(78.35,91.8)$ \\
CI 95\% OR & - & $2.57, \boldsymbol{p}<\mathbf{0 . 0 0 1}$ & $3.11, \boldsymbol{p}<\mathbf{0 . 0 0 1}$ \\
\hline
\end{tabular}




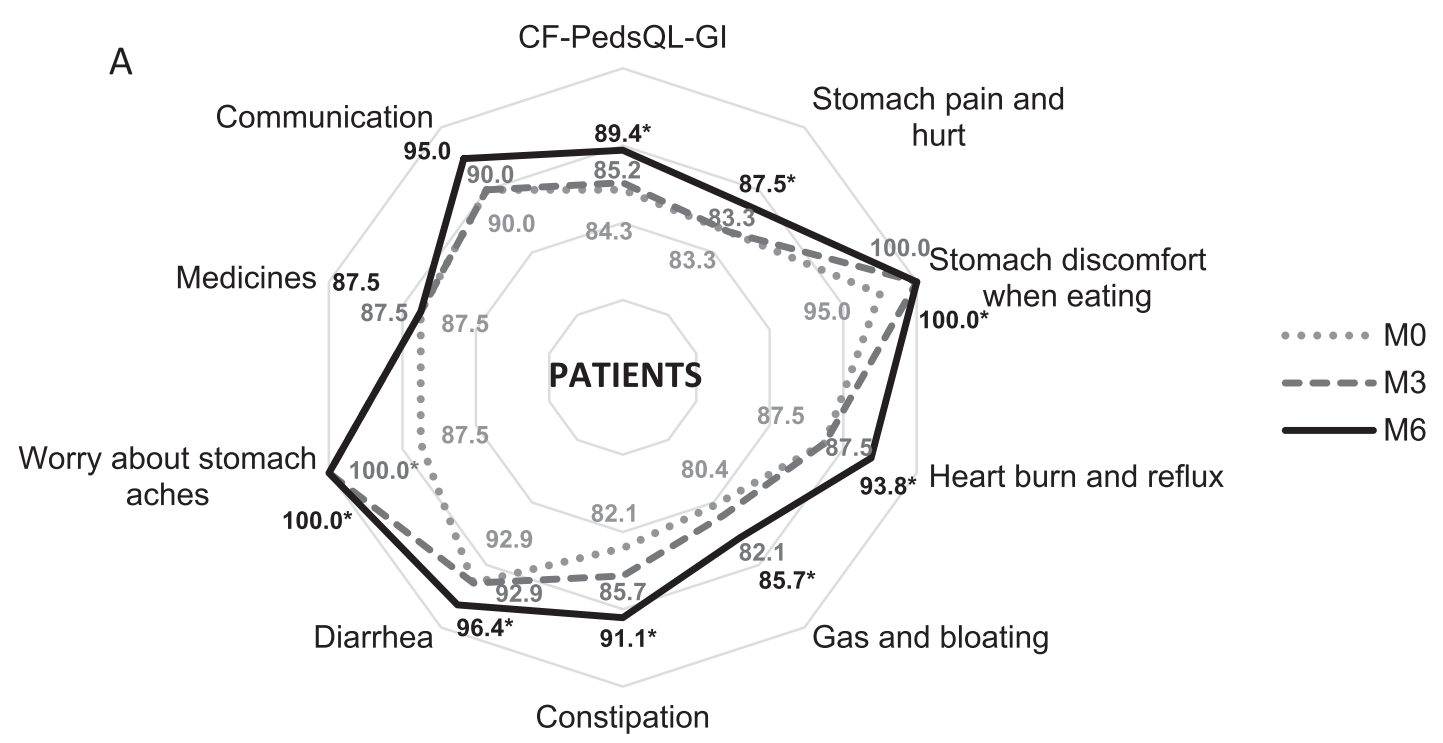

B

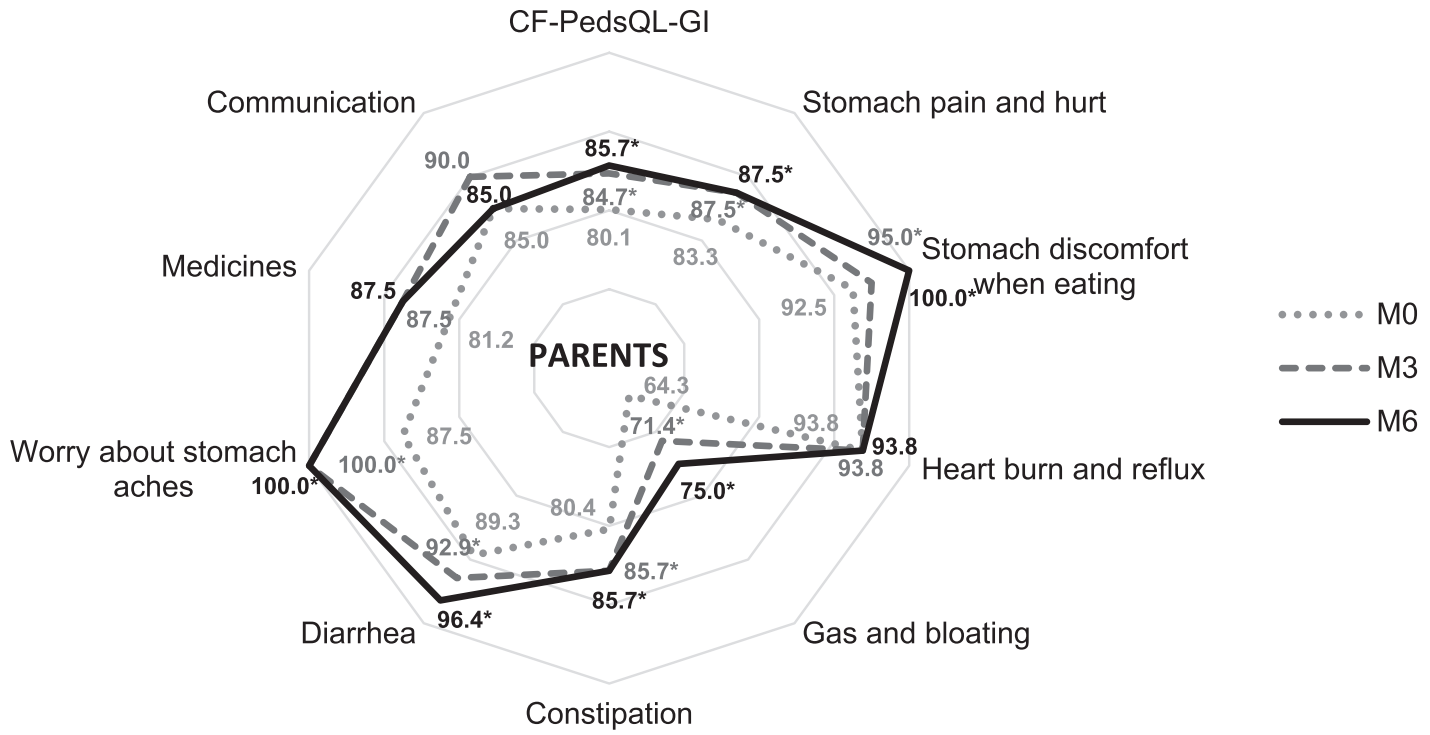

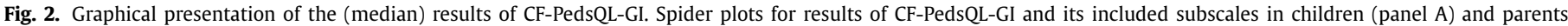

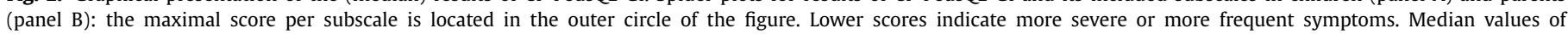

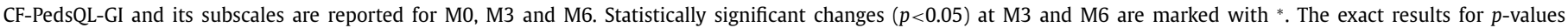
and confidence intervals for comparisons between M0 and M3/6 can be read in Supplementary files 6 and 7 .

Statistically significant changes were seen within individual centers: an increase in Lisbon and Madrid, and a decrease in Leuven (Supplementary file 14).

Higher\% of app use was associated with an increase of PERT dose (effect size $0.28,95 \% \mathrm{CI}[0.039,0.523], p=0.022$ ).

\subsection{Multivariable analyses of CF-PedsQL-GI with co-variables}

\subsubsection{Multivariable longitudinal analysis: CF-PedsQL-GI}

Change over time was associated with CF-PedsQL-GI at M3 $(\mathrm{OR}=1.68, p=0.03)$ and at $\mathrm{M} 6(\mathrm{OR}=3.19, p<0.001)$ in patients.\% App use was not associated with CF-PedsQL-GI $(p=0.51)$. Positive associations were found between CF-PedsQL-GI and age $(\mathrm{OR}=1.05$, $p=0.007)$ and baseline $\mathrm{FEV}_{1} \mathrm{z}$-Score $(\mathrm{OR}=1.03, p=0.035)$. No association was detected with gender ( $\mathrm{p} 0.06$ ) or with baseline BMI z-Score $(p=0.09)$.
3.4.2. Multivariable analysis: change in CF-PedsQL-GI after 6 months Baseline CF-PedsQL-GI was associated with change in CFPedsQL-GI; a lower baseline CF-PedsQL-GI resulted in a higher increase $(+0.54)$ in CF-PedsQL-GI after 6 months. Change in CFPedsQL-GI did not correlate with age, gender and FEV $_{1}$ z-Score.

\subsection{Post-hoc power analysis}

CF-PedsQL-GI increased by 3.8 units rather than the anticipated 3.5. The number of patients included was lower than planned (171 and not 200). However, a post-hoc power analysis demonstrated a power of $81.2 \%$ for a sample size of 150 .

\section{Discussion}

This study assessed the influence of a tailor-made mobile app for PERT self-management in CF (MyCyFAPP) on GI QOL. It is the 
first large multicentre study to evaluate the use of an app, implementing the first evidence-based method to adjust PERT $[8,9]$ based on in vitro digestion studies in CF specific circumstances.

We found a significant and promising improvement in GI QOL, measured by change in CF-PedsQL-GI. These findings are relevant, as patients rate treatment burden and GI symptoms very high on the list of research priorities [7]. Despite difficulties with recruitment, mainly due to competition with clinical trials involving CFTR modulators in the participating centers, power was reached, and the improvement of GI QOL was even higher than anticipated. The levels of CF-PedsQL-GI at the end of the trial were very close to healthy control values (around 90\%), with ceiling effects for several subscales [22].

We observed a sustained increase in CF-PedsQL-GI at M6 and \% app use as requested (46\%, where we had only aimed at $40 \%$; daily during the first month, twice weekly from the second month). This finding suggests patients and parents perceived a benefit from using the app, outweighing the potential burden. Not using the app with every meal could potentially have underestimated the effect of using the enzyme calculation module. But, this $46 \%$ app use doesn't take into account the learning effect of using the app: patients remembered the PERT doses needed for frequently consumed meals and consequently used the app for new/unknown meals by the end of the study. Positive results in terms of QOL are in accordance with the user experience as assessed by means of interviews with patients and parents [25]: most parents and patients were keen to use the app in the future.

Some results require more research. First, contradictory changes in height and weight were observed. Normal growth would result in no change in median height z-score, and therefore the observed median increase in height $z$-score during the study $(+0.13)$ may reflect an overall improvement in health. In contrast, the decrease in weight $z$-score $(-0.06)$ is small and can potentially be attributed to disease-unrelated factors (fasting for blood sampling, timing related to meals, clothing influenced by season and weather conditions). Keeping in mind the resulting decrease in BMI z-score $(-0,28)$, we cannot draw conclusions. Height and weight measurements were not standardized, and might therefore be inaccurate. Further long-term studies would need to evaluate the long-term effect of using the app on growth. Second, overall PERT dose did not change during the study, although individual centers adapted their prescription rates (some increased, others decreased), thus advising a better match between individual meals and PERT doses. A more uniform use of PERT doses among centers could be a reason for improved QOL despite unchanged median PERT doses. Thirdly, the use of the questionnaires to evaluate GI QOL might be controversial. We decided not to use CFQ-R, the most commonly used CF-specific QOL questionnaire, as primary outcome. Within the MyCyFAPP project the CF-PedsQL-GI was validated for use in children with CF focusing on GI symptoms [15]. The present results demonstrate its responsiveness to an intervention. Likewise, the CFAbd-Score, a newly developed and validated questionnaire to evaluate GI QOL in CF, [17-19] was responsive to the intervention as the score improved statistically significantly.

A limitation of the study is the lack of a control group, as it would have been ideal to include a control group with care as usual. This was not achievable due to the expected small samples in each participating center. However, we did obtain data on natural variability of CF-PedsQL-GI before [15], and the present increase of CF-PedsQL-GI exceeded this natural variability. Ideally, future research will include a control group, to confirm the findings that suggest an increase in QOL associated with using the app.

Another limitation is the inability to determine which specific part of the app was crucial for improvement of GI QOL (PERT prediction, educational content, PWT, symptoms registration module,...). Most patients/parents did state that the enzyme calculation module and nutrition follow-up had been the most useful features [25]. The burden of using the app was limited, as no patient discontinued for that reason and a considerable number of patients wanted to continue using the app after ending the trial.

The strengths of this study relate to the custom-made medical device resulting from the co-creation process with final users, the novel and scientifically pre-validated method to adjust PERT, and the innovative concept of PERT adjustment to every possible meal at any time. Implementation of the MyCyFAPP may support self-management regarding diet adjustment and PERT intake, potentially also resulting in improvement in nutritional parameters.

In conclusion, we clinically validated the MyCyFAPP, as first mobile health support for self-management of PERT in children with $\mathrm{CF}$. Implementation of the app in the day to day clinic within the context of a clinical trial was associated with an improvement in GI QOL, with few adverse events. Further steps will include further confirmation of the positive effect of using the APP on QOL, and hopefully obtaining a CE Mark certification for the MyCyFAPP as well as its implementation in regular clinical practice.

\section{Credit Author Statement}

- Mieke Boon: Conceptualization, Methodology, Formal analysis, Investigation, Resources, Data Curation, Writing - Original Draft, Writing - Review \& Editing, Visualization

- Joaquin Calvo-Lerma,: Conceptualization; Methodology; Software, Formal analysis, Investigation, Resources, Data Curation, Writing - Original Draft, Writing - Review \& Editing, Visualization, Project administration

- Ine Claes: Investigation, Resources

- Trudy Havermans: Conceptualization; Methodology, Resources, Writing - Original Draft, Writing - Review \& Editing

- Ines Asseiceira: Investigation, Resources

- Anna Bulfamante: Investigation, Resources

- Maria Garriga: Methodology, Investigation, Resources

- Etna Masip: Investigation

- Bo A.M. van Schijndel: Investigation, Resources

- Victoria Fornes: Software, Formal analysis, Resources, Data Curation, Writing - Review \& Editing, Visualization

- Celeste Barreto: Methodology, Investigation

- Carla Colombo:; Methodology, Investigation

- Paula Crespo: Software, Investigation

- Saoia Vicente: Investigation

- Hettie Janssens: Methodology, Investigation, Writing - Review \& Editing

- Jessie Hulst: Methodology, Investigation, Writing - Review \& Editing

- Peter Witters: Methodology, Investigation

- Rita Nobili: Investigation, Resources

- L.uisa Pereira; Methodology, Investigation

- Mar Ruperto: Investigation, Resources

- Els Van der Wiel: Methodology, Investigation, Resources

- Jochen G. Mainz: Conceptualization, Resources

- Kris De Boeck: Conceptualization; Methodology, Investigation, Resources, Writing - Original Draft, Writing - Review \& Editing, Supervision

- Carmen Ribes-Koninckx: Conceptualization; Methodology, Resources, Writing - Review \& Editing, Supervision, Project administration, Funding acquisition

\section{Declaration of competing interest}

All authors report grants from the European Commission Horizon 2020 during the conduct of the study. Dr. Mainz reports grants and personal fees from Vertex Corp., during the conduct of the study; Dr. Janssens reports grants from Vectura, personal fees from 
Vertex, outside the submitted work; Dr. Boon reports grants from University Hospital Leuven, outside the submitted work; Dr. De Boeck reports other financial support from Boehringer, Protalix, Raptor, Teva, Novabiotics, Eloxx, Vertex, Galapagos, Chiesi, outside the submitted work. Several authors of this publication are member of the European Reference Network for Rare Respiratory Diseases (ERN-LUNG) - Project ID No 739546.

\section{Acknowledgments}

We thank all participating children and their parents.

We thank all the participants of the MyCyFAPP consortium, especially the technical partners for the development of the APP and the games.

We thank Dr. Anke Jaudszus for her help to include the CFAbdScore as a secondary outcome measure.

\section{Supplementary materials}

Supplementary material associated with this article can be found, in the online version, at doi:10.1016/j.jcf.2020.04.001.

\section{References}

[1] Gaskin KJ. Nutritional care in children with cystic fibrosis: are our patients becoming better? Eur J Clin Nutr 2013;67:558-64.

[2] Layer P, Kashirskaya N, Gubergrits N. Contribution of pancreatic enzyme replacement therapy to survival and quality of life in patients with pancreatic exocrine insufficiency. World J Gastroenterol 2019;25(20):2430-41 28.

[3] Smyth AR, Bell SC, Bojcin S, Bryon M, Duff A, Flume P, et al. European cystic fibrosis society standards of care: best practice guidelines. J Cyst Fibros 2014;13(Suppl 1):S23-42.

[4] Turck D, Braegger CP, Colombo C, Declercq D, Morton A, Pancheva R, et al. ESPEN-ESPGHAN-ECFS guidelines on nutrition care for infants, children, and adults with cystic fibrosis. Clin Nutr 2016;35(3):557-77.

[5] Singh VK, Schwarzenberg SJ. Pancreatic insufficiency in cystic fibrosis. J Cyst Fibros 2017;16(Suppl 2):S70-8

[6] Calvo-Lerma J, Hulst JM, Asseiceira I, Claes I, Garriga M, Colombo C, et al. Nutritional status, nutrient intake and use of enzyme supplements in paediatric patients with cystic fibrosis; a European multicentre study with reference to current guidelines. J Cyst Fibros 2017;16(4):510-18.

[7] Rowbotham NJ, Smith S, Leighton PA, Rayner OC, Gathercole K, Elliott ZC, et al. The top 10 research priorities in cystic fibrosis developed by a partnership between people with $\mathrm{cf}$ and healthcare providers. Thorax 2018;73(4):388-90.

[8] Simmonds NJ, MacNeill SJ, Cullinan P, Hodson ME. Cystic fibrosis and survival to 40 years: a case-control study. Eur Respir J 2010;36(6):1277-83.

[9] Calvo-Lerma J, Martinez-Jimenez CP, Lázaro-Ramos J-P, Andrés A, Crespo-Escobar P, Stav E, et al. Innovative approach for self-management and social welfare of children with cystic fibrosis in Europe: development, validation and implementation of an mHealth tool (MyCyFAPP). BMJ Open 2017;7(3):e014931.
[10] Calvo-Lerma J, Fornés-Ferrer V, Peinado I, Heredia A, Ribes-Koninckx C, Andrés ABlachier F, editor. A first approach for an evidence-based in vitro digestion method to adjust pancreatic enzyme replacement therapy in cystic fibrosis. editor. PLoS One 2019;14(2):e0212459.

[11] Calvo-Lerma J, Hulst J, Boon M, Colombo C, Masip E, Ruperto M, et al.Blachier $\mathrm{F}$, editor Clinical validation of an evidence-based method to adjust pancreatic enzyme replacement therapy through a prospective interventional study in paediatric patients with cystic fibrosis. editor. PLoS One 2019;14(3):e0213216.

[12] Varni JW, Kay MT, Limbers CA, Franciosi JP, Pohl JF. PedsQL gastrointestinal symptoms module item development: qualitative methods. J Pediatr Gastroenterol Nutr 2012;54(5):664-71.

[13] Varni JW, Bendo CB, Denham J, Shulman RJ, Self MM, Neigut DA, et al. PedsQL gastrointestinal symptoms module: feasibility, reliability, and validity. J Pediatr Gastroenterol Nutr 2014;59(3):347-55.

[14] Boon M, Claes I, Havermans T, Fornés-Ferrer V, Asseiceira I, Bulfamente A, et al. WS12.6 mycyfapp project: validation of the PedsQL GI symptom scale to evaluate gastro-intestinal symptoms in children with cystic fibrosis. J Cyst Fibros 2018;17:S23.

[15] Boon M, Claes I, Havermans T, Fornes V, Calvo-Lerma J, Asseiceira I, et al. Assessing gastro-intestinal related quality of life in cystic fibrosis: validation of PedsQL GI in children and their parents. PLoS One 2019;14(12):e0225004.

[16] Quittner AL, Sawicki GS, McMullen A, Rasouliyan L, Pasta DJ, Yegin A, et al. Psychometric evaluation of the cystic fibrosis questionnaire-revised in a national sample. Qual Life Res 2012;21(7):1267-78.

[17] Tabori H, Arnold C, Jaudszus A, Mentzel H-J, Renz DM, Reinsch S, et al.Latzin P, editor Abdominal symptoms in cystic fibrosis and their relation to genotype, history, clinical and laboratory findings. editor. PLoS One 2017:12(5):e0174463.

[18] Tabori H, Jaudszus A, Arnold C, Mentzel H-J, Lorenz M, Michl RK, et al. Relation of ultrasound findings and abdominal symptoms obtained with the CFAbd-score in cystic fibrosis patients. Sci Rep 2017;7(1):17465.

[19] Jaudszus A, Zeman E, Jans T, Pfeifer E, Tabori H, Arnold C, et al. Validity and reliability of a novel multimodal questionnaire for the assessment of abdominal symptoms in people with cystic fibrosis (CFAbd-score). Patient- Patient-Centered Outcomes Res 2019;12(4):419-28.

[20] Floch J, Zettl A, Fricke L, Weisser T, Grut L, Vilarinho T, et al. User needs in the development of a health app ecosystem for self-management of cystic fibrosis: user-centered development approach. J Med Internet Res 2018;6(5):e113.

[21] Vilarinho T, Floch J, Stav E. Co-designing a mHealth application for self-management of cystic fibrosis. Lect Notes Comput Sci (including Subser Lect Notes Artif Intell Lect Notes Bioinformatics) 2017;10515:3-22.

[22] Varni JW, Franciosi JP, Shulman RJ, Saeed S, Nurko S, Neigut DA, et al. PedsOL gastrointestinal symptoms scales and gastrointestinal worry scales in pediatric patients with inflammatory bowel disease in comparison with healthy controls. Inflamm Bowel Dis 2015;21:1115-24.

[23] Kuczmarski RJ, Ogden CL, Guo SS, Grummer-Strawn LM, Flegal KM, Mei Z et al. CDC growth charts for the United States: methods and development. nationale center for health statistics. . Vital Health Stat 2000;2002(246):1-190.

[24] Ouanjer PH, Stanojevic S, Cole TJ, Baur X, Hall GL, Culver BH, et al. Multi-ethnic reference values for spirometry for the 3-95-yr age range: the global lung function 2012 equations. Eur Respir J 2012;40(6):1324-43.

[25] Floch J, Vialrinho T, Zettl A, Ibánez G, Calvo-Lerma J, Stav E, et al. JMIR Preprints \#15896: User Experience of a mHealth Self-Management Approach for the Treatment of Cystic Fibrosis [Internet]. [cited 2019 Nov 15]. Available from: https://preprints.jmir.org/preprint/15896. 